\title{
The Lens MetaRecord and LensID: An open identifier system for aggregated metadata and versioning of knowledge artefacts
}

\section{Working paper}

Osmat A. Jefferson ${ }^{a}$, Deniz Koellhofer, Ben Warren, and Richard Jefferson

Queensland University of Technology \& Cambia.

${ }^{a}$ Corresponding author.

\begin{abstract}
Ambiguity is inherent in the digital records of entities such as patents, scholarly works, human names, or institutions. While we have made some progress to preserve each entity's one to one relationship using open persistent identifiers, in this contribution, we show how the Lens has used the MetaRecord (MeR) concept along with the open LensID identifier to begin mapping the one to many relationships among these data elements, disambiguating name variants, and organizing contextual metadata.
\end{abstract}

\section{Introduction}

Our world is different now. Increasingly more connected via digital decision analyses and communications, yet when linking data elements in an innovation ecosystem to help solve a problem, one is faced with an overwhelming confusing mess. Most information is still stored in silos and if accessible, often machine unreadable, ambiguity in humans or institutions names is high, and variation in recorded knowledge across various sites is confusing. This structural complexity generates inefficiencies, errors, and uncertainty in the innovation sector.

Besides conferring an exclusive right on an invention or a solution to a problem, a patent is an accumulated body of scientific, technical, and industrial knowledge that is continuously evolving based on other related knowledge artefacts. To gain insight on an invention in an innovation pathway, it is critical to access, analyse, and monitor its varied contextual metadata; the patent file wrapper, its family members, the citations, the office actions, the legal challenges, and other relevant data elements.

While patent offices provide information retrieval systems based on patent publication key numbers, mapping capabilities of the contextual complexity around an invention to improve

\footnotetext{
${ }^{1}$ Copyright $\odot 2019$ by Lens.org

Working papers are in draft form. This working paper is distributed for purposes of comment and discussion only. It may not be reproduced without permission of the copyright holder. Copies of working papers are available from the author.
} 
one's confidence level is still lacking. Similarly, with the recent proliferation of scholarly preprints, postprints, annotations, commentary, and other related data, many public institutions struggle to implement and provide library users with their own comprehensive metadata on scholarly works.

To enable a clearer and deeper understanding of the diverse capabilities necessary for science and technology to effectively and efficiently contribute to solving problems of critical importance to society, The Lens has begun addressing some of the encountered structural complexities around innovation knowledge artefacts (KA), initially patents and scholarly works.

Currently, the Lens hosts more than $118 \mathrm{M}$ patent and $208 \mathrm{M}$ scholarly work records, representing at least 95 jurisdictions and 193 affiliation-based countries, respectively. Moreover, the Lens enriches its biological data with more than $313 \mathrm{M}$ disclosed genetic sequences and their metadata. All Lens data is served as annotatable digital public goods resolvable by identifiers.

In addition, The Lens implements a MetaRecord (MeR) concept to manage complexities around record variability, sources and contextual metadata relevance to the original record. Complemented with a unique open persistent identifier, LensID, The Lens MetaRecord reflects an open, granular, and dynamic mapping system with a logging history of knowledge around a knowledge artefact entity. Such entity can be a patent, a scholarly work, a human name, or an institution. In this contribution, we describe the concept and its applications in patents and scholarly works, and discuss its interoperability with other identifiers and potential use in disambiguation of human and institution names.

This concept is similar to the thoroughly reviewed concept of "work" among cataloguers in libraries with successfully complex bibliographic retrieval tools (Smiraglia, 2001). Leading librarians such as Antonio Panizzi, Eva Verona, Seymour Lebetzky and Kristin Antelman have actually shaped that concept as a "central object for retrieval" in the bibliographic universe to exploit recorded knowledge and over time, there seems to be a consensus that while relationships among works are complex, a taxonomy of relationships can still be structured to allow for explicit expression for information retrieval (Antelman, 2004). Antelman advanced the need for a "superwork record"identifier that focuses on functional relationships among various editions, and variation in the author names, bibliographic information of a work rather than its descriptive details and highlighted the need for such a system to preserve and enrich users access and use of collective knowledge on a work. The Lens Metarecord and its LensID aspires to provide such a solution.

\section{MetaRecords for Patents and Scholarly works}

Patents, scholarly works, and their related documents occur in myriad forms and locations. In addition, variation in the digital archiving of records can be overwhelming and hard to capture, standardise and disambiguate (Figure 1). While a canonical version of a scholarly 
work can be linked to the publisher's website via a digital object identifier (DOI)-based url (DOI Factsheet, 2019), if a publisher ceases to exist, the link breaks or if there are other versions of the scholarly work (submitted version, preprint, translations, etc..) or associated metadata (eg. citations in patents or scholarly works, or on social media), these can not be readily accessed or linked to using this identifier alone, and users of that innovation information would miss on the context, meaning and utility of such work.

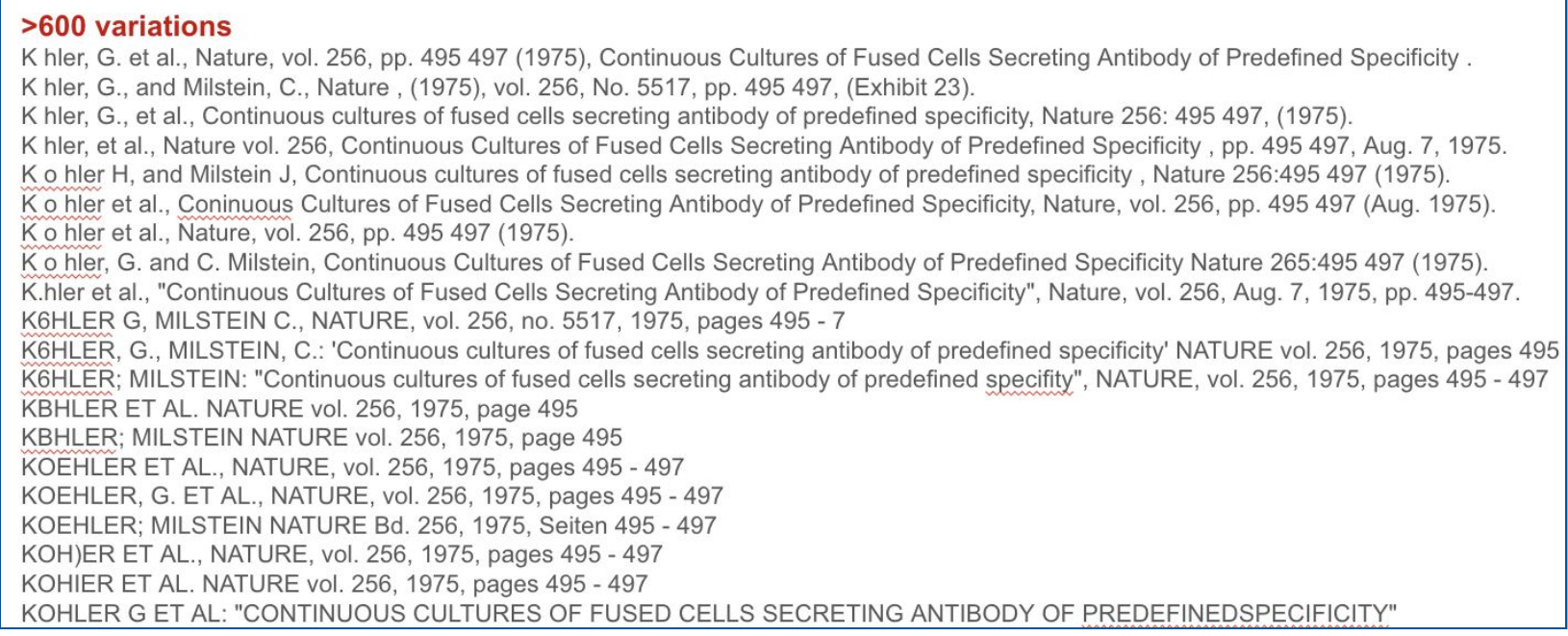

Figure 1. A sample of the 600 variations found in the name of authors/digital archiving of this scholarly work.

Similarly, as patent documents are not copyrighted, they are often present on numerous websites in varying degrees of utility- as XML, PDFs, text files, with or without associated files such as sequences, chemical structures or equations, with or without necessary citations and legal status. The legally enforceable form of a patent can be a TIFF image file of the official document (such the case in the USA), however, this image file, is rarely a useful artefact except in a court of law. Every patent offers a complex history of negotiation and clarification between inventor (or her counsel) and the patent office, in the form of a file-wrapper, or prosecution history that is often essential to provide agreed definitions, contextual or prior art, and clarifications of the teachings.

To capture the constellation of related and contextual data and aggregate versions and source URLs for a knowledge artefact such as a patent or a scholarly work record and enrich innovation community user experience, the Lens has deployed a broadly-defined and evolving MetaRecord that is discoverable and resolvable by a new unique open persistent identifier, LensID. The Lens MetaRecord aggregates and normalizes much of what we or others can harvest about that patent or scholarly record and which can then be publicly discoverable and available for verification by third parties.

In the Lens, the scholarly work LensID 024-987-588-745-109 captures related versions and variants on that work including associated metadata on scholarly and patent citations, mode and type of access along with licensing information and renders that information publicly 
accessible and available for public scrutiny. Moreover, the patent record with LensID lens.org/029-768-644-186-340 includes the legal form of the patent document (the image file) and the entire ecosystem of supported knowledge, including filing history, inventor attested ID, if available, citations, associated sequence listing files, and legal status.

An important aspect of Lens MetaRecords is their granularity. A Lens MetaRecord is not fixed. It can evolve and improve as new sources of the knowledge artefact and associated metadata become available. Thus, they serve *not ${ }^{\star}$ just as a replacement for any author or institution's version, but rather as a dynamic, open linking, harvesting, and cataloguing tool that optimizes the quality and utility of knowledge around an artefact.

Maintaining provenance of metadata and keeping a log for changes is a key for such an aggregative MetaRecord, especially in name disambiguation projects. Figure 2 displays how ID clusters get formed and edited when supplementary data is supplied in the backend of the Scholarly Meta-Record data store.

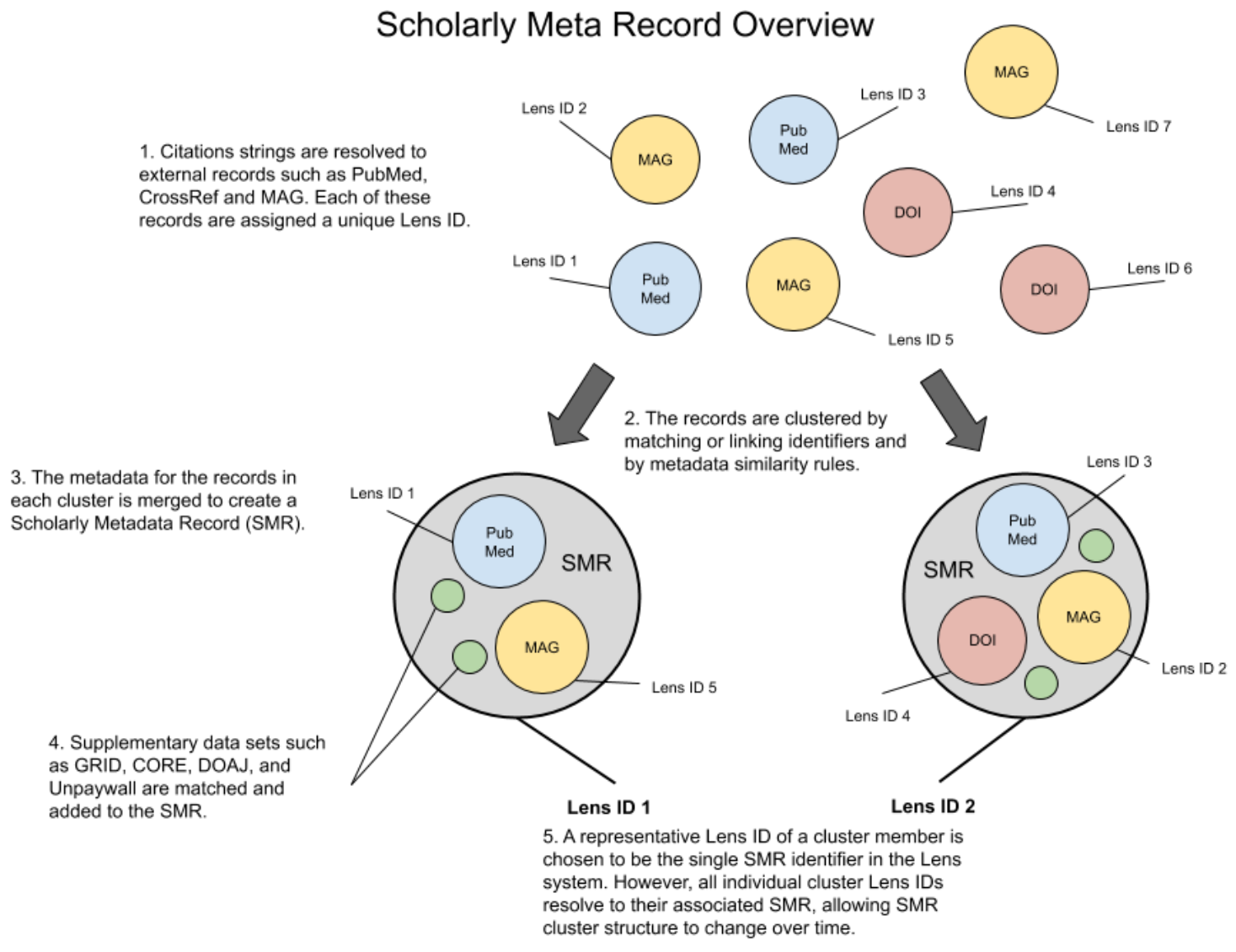

Figure 2. The Lens Scholarly Meta-Record (SMR) modeller. 


\section{Format of LensID}

As with other open persistent identifiers, the LensID uses a purely numeric field without any internal business logic such as patent jurisdiction or scholarly work publisher's name. Moreover, the number is assigned randomly along with a checksum for quality control. The numerical structure of LensID was developed with valuable input from Geoffrey Bilder and in collaboration with Crossref (Bilder, 2012). Informed by the non profit organization ORCID and the International Standard Name Identifier (ISNI) schemes and to avoid any clashes or confusion with them, we have used a 15 rather than 16 digit identifier with the following features:

1. The 15 digits long Lens identifier is broken in 5 groups where every 3 rd digit is separated from the next with a dash (-).

2. The last digit is a checksum digit which helps detect copying errors. The check digit can have values $0-9$ and ' $X$ ' where $X$ represents a value of 10 (check the code to generate the checksum digit).

3. The check digit is calculated using mod the 11,2 algorithm outlined in The International Organization for Standardization (ISO) standard 7064.

4. The Lens can resolve LensIDs via URLs of the form https://lens.org/[LensID] , where [LensID] is replaced with the LensID to resolve. For example, the LensID 192-920-770-759-49X can be resolved via the URL https://lens.org/192-920-770-759-49X fitting the description of a persistent identifier and its associated "descriptive landing page" for a resource.

5. Lens identifiers are randomly generated and their uniqueness verified for allocation to resources. Being opaque and from a large value space, LensIDs are highly granular as they could be assigned to any data type.

6. The identifiers point to a particular resource and always will. As they are not categorised or partitioned by business rules in any way, changes to the business logic within the Lens will never affect identity.

7. LensIDs can be efficiently stored and used as a numeric value by dropping the check digit. For example, 192-920-770-759-49X becomes 19292077075949.

8. Other identifier types (Patent IDs, DOIs, PMIDs, etc) can be found within the LensID records themselves. These are looked up by the Lens resolver service using the LensID and returned to direct the user to the appropriate record in the system.

We provide the structure of the LensID in Figure 3. 


\section{LensID_15 digit congruent with ORCID and ISNI schemes}

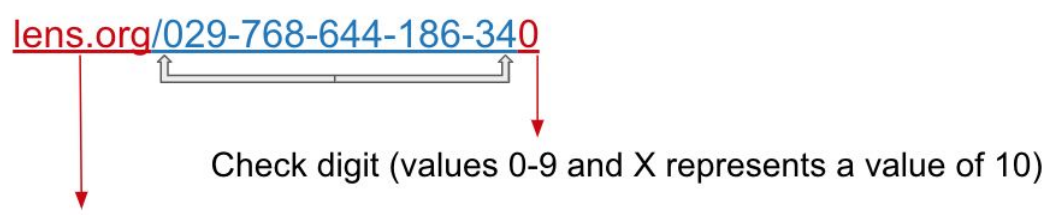

Prefix. The hyperlink should point to https://lens.org/029-678-644-186-340 and this applied to URLs in web service data as well. Using HTTPS allows the user to privately request documents from lens.org

Figure 3. Depiction of the LensID structure.

\section{Interoperability with external scholarly identifiers}

Realizing that both inventions and discoveries have at their core the generative role of individuals and that inventors themselves are often in the private sector, and may be largely 'invisible' to the world of academic practice, the Lens is also facilitating the attestation of inventors work products and interoperability of identifiers across communities. Even if a user is not a listed inventor on a patent record, we think that there is much to be gained from linking a LensID to an ORCID iD for that person.

The Lens brings both scholars and inventors into the ORCID iD system by enabling ORCID users to search the Lens, and using OAuth protocol and their ORCID Account login credentials, mark-up and associate patent inventorship with their profile. If their Lens account is linked to their ORCID record and inventorship is claimed, then in their ORCID record, their patent "Work" records will be automatically recorded with the source set to 'The Lens". Claiming inventorship ensures that proper credit can be given for being an inventor on a published patent, patent application or plant or design patent. It also helps in a global effort to disambiguate inventors with similar names.

We think it is important that the 'crossover' role of many scholars and inventors is recognized, and the role of scholars in contributing to the innovation system as measured by patents, is explored.

With Crossref, PubMed at NIH, CORE, and Microsoft Academic, we have extracted and integrated their scholarly work datasets into the Lens as well as matched and linked non-patent citation strings to DOIs, PubMed IDs (PMIDs), and MAG IDs within patents and through the use of CORE and Unpaywall databases, identified Open Access scholarly works and linked to the full-text version. In the Lens, users can now connect to the various sources of any scholarly data and metadata via the multiple external identifiers that the Lens exposes on site. 
Scholarly works, which are cited by inventions, are important 'prior art' or enable disclosures. With only a few clicks, an ORCID or a Linkedln account holder with a list of publications can now find the world's patents that have cited their scholarship and link to these in the Lens.

\section{Extending the Lens MetaRecord to humans and Legal entities}

The proposition of having identifiers for inventors can be a substantial contribution to improving clarity in global patenting, however, considering that inventors often have no real interest in the patent publication, either in professional credit or financial outcomes and do not typically have control over the patent document or its rights, the way authors of scholarly publications and grants do, makes such exercise inefficient. Patents are rather written often by patent attorneys in consultation with inventors, and the employer - generally a company or institution - has the resulting rights and manages licensing and negotiation with the patent office, including paying the ongoing costs.

During the course of 'prosecuting' or progressing a patent through the filing and examination process, the patent office enters into a contract with the applicant/owner as their customer; it is almost never the inventor who is the owner. There is a contrived circumstance in the USA, in which, for compliance with the law, the applicant at the moment of filing must be an inventor, the rights are almost always assigned immediately to their employer as a consequence of their employment contract. Thus the patent office would have to require the assignee (applicant) to, in turn, require that the inventor(s) (who typically but not always are employees) create and manage an identifier. This indirect relationship with an inventor makes it much less likely to be implemented than the case with granting agencies or publishers, in which there is a direct contract with the author, and there are direct and substantial 'carrots' to be held out for compliance, and sticks for non-compliance.

To mitigate these issues, The Lens proposes the use of the LensID MetaRecord concept to organise knowledge on inventors, including their names. If we just consider the ambiguity or similarity in inventors' names from patents and authors' from Scholarly records, one can recognize the potential multidimensional functional relationships that can exist among such names based on their relevance to patent or scholarly works. Tracking and exploiting this relevant information will require a move beyond a one-to one relationship identifier system and more towards a log system that exposes these relationships at multi- levels and enables modular mapping of the one to many relationships.

With the help of a LensID, for example, we can capture and cluster all name variations to a new LensID. Then using such LensID as an anchor, we build an open map that could be publicly verified and checked for accuracy. And because each algorithm/stage of the mapping is reversible, The Lens can then enable users to group IDs while keeping track of such optimizations for others. This will also allow mapping of more complex queries based on the integration of intermediates LensIDs in the searches. 
The critical part of the open mapping strategy is cascading initial LensIDs onto subsequent IDs, with a full logged map history. No ID is reused.

Figure 3 illustrates a simple example where in one finds that in the patent literature, there are 18 inventors or applicants named "Richard Jefferson" and based on the contextual information, they are likely to be the same inventor, then these will be clustered and mapped to a new LensID (Figure 4), and the mapping will be logged. Subsequently, if there are 43 Lens IDs for "Richard A Jefferson" as authors in the scholarly space, and some are found likely to be the same inventor of Richard Jefferson in the patent space, then grouping and mapping those across patent and scholarly datasets onto a new LensID will enrich further information discoverability and retrievability and remove confusion on this name.

\section{Example of open action-history logging of identifiers}

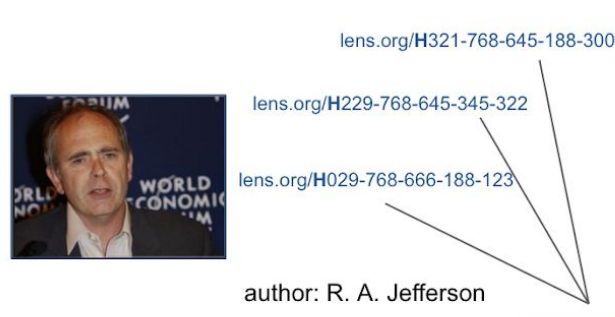

author: R. A. Jefferson

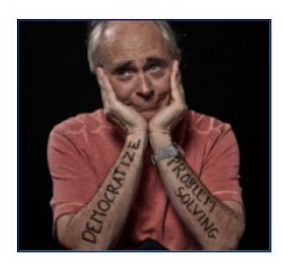

lens.org/H029-768-645-188-200

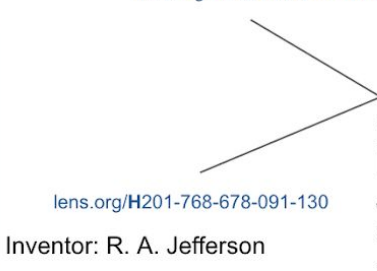

lens.org/H302-700-555-188-200

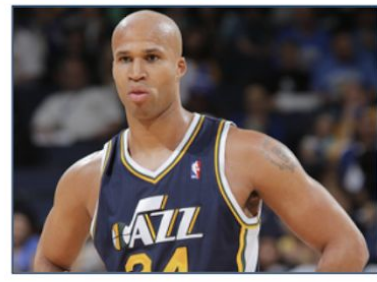

Author: R. A. Jefferson

lens.org/H021-455-644-222-868

New Lens ID is generated to cluster the Inventor and author name of Richard Jefferson based on the mapping of knowledge artifacts associated with this name while maintaining a logging history.
lens.org/H999-547-555-987-200

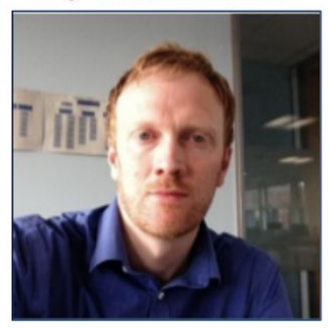

Inventor: R. Jefferson

Figure 4. Example of open action-history logging of LensIDs.

\section{Conclusion}

The proposed logging system of the LensIDs maintains the PID paradigm described earlier (Van de Sompel et al., 2014), however, like others who have recognised the need for a more decentralized management approach for persistent identifiers (Bolikowski et al., 2015), the new Lens MetaRecord concept proposes the use of identifiers as aggregators of contextual knowledge artefacts around an entity. The Lens has demonstrated its use for patent and a scholarly work records and will apply it in the near future to humans and legal entities.

\section{Acknowledgments}


This work was funded by the Bill \& Melinda Gates Foundation and the Queensland University of Technology. We thank Geoffrey Bilder and Karl Ward for their valuable inputs in the early discussions on the LensIDs. An earlier version of this work was submitted and discussed at "Identifiers and Intellectual Property" Workshop, organized by ORCID, Crossref, and OECD in Paris on June 22, 2017 (Jefferson, 2017). We acknowledge the support of the ORCID team, in particular Laurel Haak, Alice Meadows, and Alainna Wrigley and their help to bring ORCID iD searches and other functionalities into Lens.org. We also are grateful to Kristin Antelman, head librarian at the University of California-Santa Barbara for introducing us to her 2004 publication on "superwork" identifier and other leading librarians who have been shaping the nature of "work" to enrich users' access, and use of the scholarly recorded knowledge. We thank Samuel Klein from the Knowledge Future Group at MIT for his help to bring LensID into the Wikidata community and Aaron Ballagh from the Lens team for his edits and suggestions on this work.

\section{References}

Antelman, K. (2004). Identifying the serial work as a bibliographic entity. Library Resources \& Technical Services 48 (4): 238-255. https://lens.org/051-148-349-794-677

Bilder, G. (2012). Structure of the ORCID identifier. A Whitepaper. Retrieved from https://support.orcid.org/hc/en-us/articles/360006897674-Structure-of-the-ORCID-Identifier

Bolikowski,L., Nowiński, A., \& Sylwestrzak, W. (2015). A system for distributed minting and management of Persistent Identifiers. International Journal of Digital Curation 10 (1): 280-286. https://lens.org/082-623-578-255-305

Code to generate the checksum digit is also available at https://support.lens.org/help-resources/basic-help/the-lens-id/

DOI Factsheet (2019). http://www.doi.org/factsheets/DOIKeyFacts.html

Haak, L. (2017). Identifiers and Intellectual Property: About ORCID. Presentation at Identifiers and Intellectual Property Workshop on June 22, 2017 at OECD headquarters, Paris, France. https://lens.org/107-937-945-316-534

Jefferson, O. A. (2017). Using identifiers in patent databases. Presentation at Identifiers and Intellectual Property Workshop on June 22, 2017 at OECD headquarters, Paris, France. https://lens.org/048-444-597-260-357

Jefferson, R.A. (2013) Presentation at The Skoll World Forum on Social Entrepreneurship. Retrieved from https://www.youtube.com/watch?v=9Bddt8rBgFg

LensID property is approved in Wikidata: https://www.wikidata.org/wiki/Property:P7100

Pisano, G. P. ( 2015). "You Need an Innovation Strategy." Harvard Business Review 93 (6) : 44-54. 
Smiraglia, R.P. (2001). The nature of " $a$ work", Lanham, Md: Scarecrow Press, ISBN 0810840375. https://lens.org/193-627-707-985-201

Van de Sompel, H., Sanderson, R., Shankar, H., \& Klein, M. (2014). Persistent identifiers for scholarly assets and the web: The need for an unambiguous mapping. The International Journal of Digital Curation, 9(1), 331-342. https://lens.org/036-120-861-690-596 